\title{
Somatosensory and brainstem auditory evoked potential in congenital craniovertebral anomaly; effect of surgical management
}

\author{
S Sood, A K Mahapatra, R Bhatia
}

\begin{abstract}
Clinical features and evoked potential recordings were analysed in 32 patients with congenital atlantoaxial dislocation before and after surgery. Seven patients (group 1) had atlantoaxial dislocation, while 22 patients had associated basilar invagination (group 2). In both groups, pyramidal tract signs, posterior column signs, wasting of the upper limbs, and abnormality of somatosensory evoked potentials (SSEP) were similar. Conversely, lower cranial nerve involvement and abnormal brainstem auditory evoked potentials (BAEP) were significantly more in patients with basilar invagination $(\mathbf{p}<0.05)$. All seven patients in group 1 and 17 patients in group 2 were operated upon. Clinical and electrophysiological deterioration were significant in patients with basilar invagination (group 2), following posterior fixation compared with group 1. Among the patients in group 2, who clinically deteriorated following posterior fixation, seven had transoral excision of odontoid and six of them improved both clinically and electrophysiologically. Two patients in group 2 had odontoid excision before posterior fixation, and in both the evoked potentials improved postoperatively. In group 1 the patient's BAEP remained unaffected following posterior fixation, however, in group 2, eight patients over $53 \%$ showed improvement in brainstem function following posterior fixation. This study shows the value of evoked potentials in congenital atlantoaxial dislocation, and rationalises the surgical procedure in these patients. In patients with basilar invagination, odontoid excision is the preferred first stage procedure.
\end{abstract}

Department of Neurosurgery, Neurosciences Centre, All India Institute of

Medical Sciences, New

Delhi, India

$S$ Sood

A K Mahapatra

R Bhatia

Correspondence to:

Dr Mahapatra, Departmen of Neurosurgery,

Neurosciences Centre, All

India Institute of Medical

Sciences, New Delhi, India

110029

Received 15 October 1990 and in revised form

and in revised 11 June 1991 .

Accepted 12 August 1991
Congenital abnormalities of the cranial base and the upper two cervical vertebrae produce signs and symptoms by extradural compression of the cord or the medulla. Longstanding compression invariably results in pathological changes and operative intervention to relieve the compression is often hazardous especially in congenital atlantoaxial dislocation. ${ }^{1-4}$ Recently evoked potentials have been used in various neurological problems. ${ }^{5-7}$ It has been reported that the brainstem auditory evoked responses (BAER) improve after posterior fixation or decompression in craniovertebral anomalies. $^{8}$ Intraoperative somatosensory recordings have shown improvement immediately following transoral excision of the odontoid. ${ }^{9}$

In this study we evaluated the anterior and posterior approaches to the craniovertebral anomalies using clinical and electrophysiological parameters.

\section{Methods and materials}

Thirty two patients with congenital craniovertebral anomalies were studied. Conventional radiographs of the craniovertebral region were taken including lateral views in maximum extension and flexion (figure 1). CT or water soluble contrast (Matrizamide/Iohexol (R)), myelography provided complementary evidence of soft tissue anomalies like syringomyelia and Arnold-Chiari malformation. In a few cases MRI was performed. The patients were divided into two groups. Group 1 were patients with atlanatoaxial dislocation only (figure 2 ) and group 2 were patients with atlantoaxial dislocation and basilar invagination (figure 3). Seven of the 10 patients of group 1 and 17 of the 22 patients of group 2 were operated on. Eight patients were not operated on either because they were considered a poor surgical risk or had refused an operation. All patients were maintained on cervical traction using Garderner-Wells tongs preoperatively for a period of 3-7 days. Patients who improved neurologically had a posterior fusion, using a suboccipital $\mathrm{C} 2$ fusion with stainless steel wire and onlay iliac bone graft. Patients with a compromised foramen magnum and $\mathrm{Cl}$ canal diameter had decompression of the posterior rim as well. Those patients who deteriorated neurologically after the above procedure, had transoral excision of

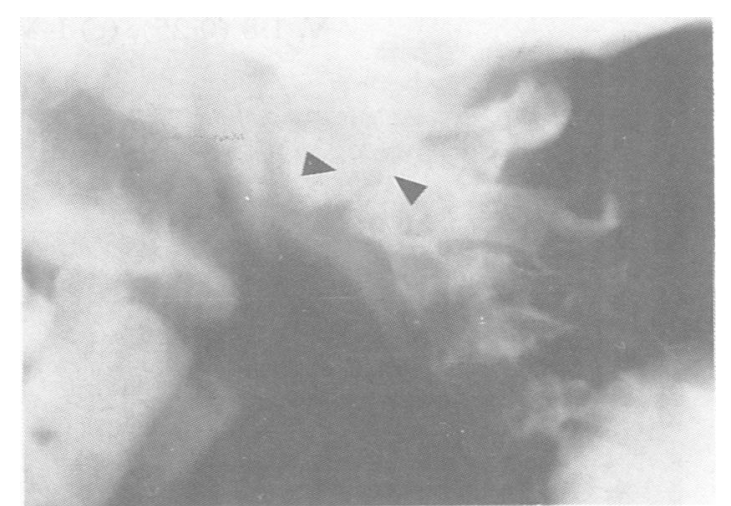

Figure 1 Plain $X$ ray lateral view of the craniovertebral junction shows atlantoaxial dislocation. 
Figure 2 Sagittal view of MRI of craniovertebral junction shows atlantoaxial dislocation but no basilar invagination.

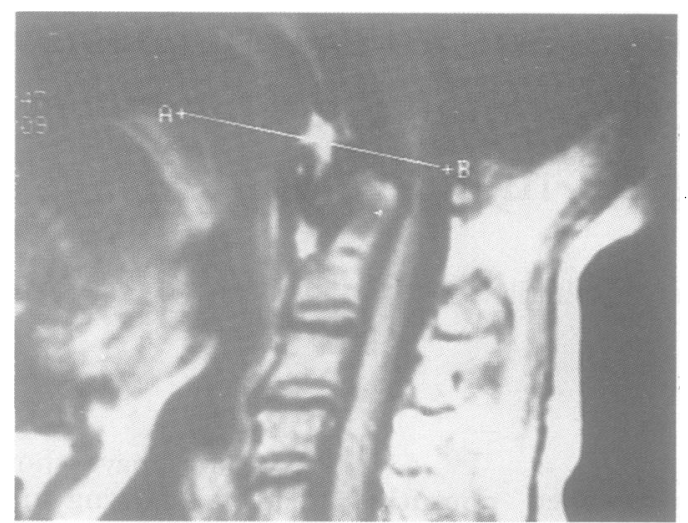

the odontoid. If neurological improvement failed to occur following traction, patients had transoral excision of the odontoid and posterior fusion.

BAEP and SSEP were recorded before cervical traction was applied and these tests were repeated on the seventh postoperative day after removal of traction. BAEP were recorded at $60 \mathrm{~dB}$ above the hearing threshold using rarefaction clicks with masking of the contralateral ear. Electrodes were placed on the mastoid tips with cephalic reference. Two thousand stimuli were averaged on the Nicolet compact four electrodiagnostic system Madison, USA. Two superimposed recordings evaluated interpeak latencies (IPL). Median nerves were stimulated with rectangular constant current pulses of $0.2 \mathrm{~ms}$ delivered at a rate of 5 a second. The intensity was adjusted to produce a twitch in the opponens pollices muscle. Evoked potentials were recorded with silver platinum disc electrodes with the resistance maintained below 5 kohms. Recordings were made at (a) the brachial plexus, active electrode at Erb's Point and reference at FZ; (b) the lower neck, active electrode at $\mathrm{C} 7$ spinous process and reference at FZ: and (c) the post-central cortex, active electrode at $\mathrm{C} 3$ $(2 \mathrm{~cm}$ behind $\mathrm{C} 3$ ) with reference at $\mathrm{FZ}$. The signals were averaged with filter setting at 5 to $1500 \mathrm{~Hz}$. At least 500 responses were averaged. Each recording was replicated and the results calculated as the average of the two recordings. The major waveforms in the median nerve recordings were labelled N9, N13, and N19.

Normal mean (SD) control values for interpeak latencies (IPL) recorded from 20 healthy individuals, were (a) I-III, $2 \cdot 12(0 \cdot 14)$, (b) III$\mathrm{V}, 1.8(0.25)$, (c) I-V, $3.9(0.2)$, (d) N13-N9,

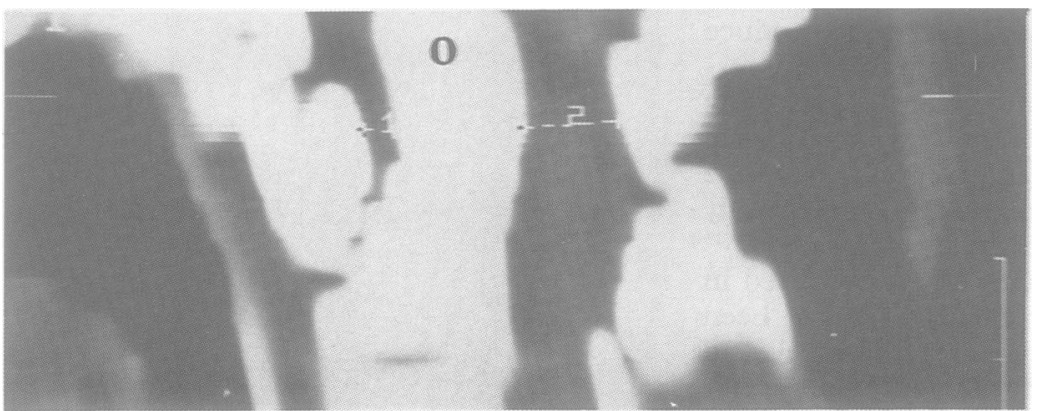

Figure $3 C T$ scan of the sagittal section through craniovertebral junction shows basilar invagination associated with atlantoaxial dislocation.
$3.44(0.43)$, and (e) N19-N9, 9.17 (0.85). IPL more than $2.5 \mathrm{SD}$ above the normal were considered abnormal and a change of more than 2 SD after surgery was considered significant at $\mathrm{p}<0.05$.

The parameters used for evaluating brainstem function were lower cranial nerve (IX, X, $\mathrm{XI})$ paresis and BAEP. Spinal cord function was evaluated using the following parameters: (a) involvement of the pyramidal tracts suggested by weakness, spasticity and extensor plantar reflex, (b) wasting of the upper limbs or abnormal N13 wave/N13-N9 IPL suggesting damage at the anterior and posterior horns respectively, and, (c) posterior column involvement present clincally or electrophysiologically (abnormal N19 wave or N19-N9 IPL). Where necessary Chi-square test with Yates correction was used to compare the two groups.

\section{Results}

In group 1, only one patient had brainstem dysfunction manifesting as lower cranial nerve palsy and prolongation of I-III IPL. There were pyramidal tract signs in eight patients, wasting of the upper limbs in five and posterior column signs in four patients. Nine patients had abnormality of N13 wave/N13-N9 IPL and in eight patients N19 wave/N19-N9 IPL was abnormal (table 1).

In group 2, five patients had lower cranial nerve palsies, two of them also had prolongation of I-III IPL. In another seven patients I-III IPL was increased. Of these patients one also had prolongation of the III-V IPL. Pyramidal tract signs were present in all patients, wasting of the upper limbs in nine and posterior column signs in 17 patients. Nineteen patients had abnormality at N13/N13-N9 IPL and 17 at N19/N19-N9 IPL. Lower cranial nerve palsies and abnormal BAEP were significantly more frequent $(3.95, p<0.05)$ in group 2 patients. The dysfunction of various tracts at the spinal cord level was not significantly different in the two groups $\left(\chi^{2} 20.51\right.$, $\mathrm{p}<0 \cdot \mathbf{1}$ ).

After posterior surgery (posterior fusion and/or decompression) in group 1, only one patient had deteriorated in one or more of the spinal cord function parameters described above (table 2). Similar deterioration occurred

Table 1 Clinical and electrophysiological features in patients of Group 1 and 2

\begin{tabular}{llc}
\hline & $\begin{array}{l}\text { Group 1 } \\
(n=10)\end{array}$ & $\begin{array}{c}\text { Group 2 } \\
(n=22)\end{array}$ \\
\hline $\begin{array}{ll}\text { Brainstem dysfunction } \\
\text { Cranial N paresis }\end{array}$ & 1 & 12 \\
I-III IPL abn & 1 & 5 \\
III-V IPL abn & 1 & 9 \\
Nystagmus & 0 & 1 \\
Spinal cord dysfunctiont & 3 & 9 \\
Wasting & & \\
N13 wave or N13-N9 IPL abn & 9 & 9 \\
Pyramidal signs & 8 & 19 \\
Posterior Column sign & 4 & 17 \\
N19 wave or N19-N9 IPL abn & 8 & 17 \\
Spinothalamic signs & 1 & 8 \\
Bladder involvement & 2 & 7 \\
\hline
\end{tabular}

*Difference between Group 1 and 2 significant at $p<0.05$ †Difference in pattern of involvement between the two groups not significant. not significant. 
Table 2 Change in the clinical and electrophysiological parameters after posterior surgery in Group 1 and 2 patients

\begin{tabular}{|c|c|c|c|c|c|c|}
\hline & \multicolumn{3}{|c|}{ Group $1(n=7)$} & \multicolumn{3}{|c|}{ Group $2(n=15)$} \\
\hline & Improved & Worsened & Unchanged & Improved & Worsened & Unchanged \\
\hline $\begin{array}{l}\text { Brainstem parameters } \\
\text { Cranial N paresis } \\
\text { I-III IPL abn } \\
\text { III-V IPL abn }\end{array}$ & $\begin{array}{l}0 \\
0 \\
0 \\
0\end{array}$ & $\begin{array}{l}0 \\
0 \\
0 \\
0\end{array}$ & $\begin{array}{l}7 \\
1 \\
7 \\
7\end{array}$ & $\begin{array}{l}8 \\
2 \\
8 \\
2\end{array}$ & $\begin{array}{l}2 \\
1 \\
1 \\
2\end{array}$ & $\begin{array}{r}5 \\
1 \\
6 \\
11\end{array}$ \\
\hline $\begin{array}{l}\text { Spinal Cord parameters } \\
\text { Wasting } \\
\text { N13-N9 IPL abn } \\
\text { Pyramidal signs } \\
\text { Posterior column signs } \\
\text { N19 wave or N19-N9 IPL abn }\end{array}$ & $\begin{array}{l}0 \\
4 \\
4 \\
0 \\
0\end{array}$ & $\begin{array}{l}1 \\
0 \\
1 \\
1 \\
1 \\
1\end{array}$ & $\begin{array}{l}5 \\
2 \\
1 \\
1 \\
6\end{array}$ & $\begin{array}{l}0 \\
6 \\
4 \\
1 \\
1\end{array}$ & $\begin{array}{r}12 \\
0 \\
7 \\
7 \\
6 \\
11\end{array}$ & $\begin{array}{l}9 \\
2 \\
4 \\
7 \\
3\end{array}$ \\
\hline
\end{tabular}

*Worsening in group 2 patients was significantly more frequent than group 1 patients at $\mathrm{p}<0.02$.

in 12 of the 15 patients in group 2 who had posterior surgery (figure 4). In 11 patients, the N19 wave/N19-N9 IPL deteriorated. In six of the 11 patients who deteriorated posterior column sensation and pyramidal signs occurred. One patient had deterioration of pyramidal signs only (table 2 ). The difference in the number of patients who deteriorated after posterior surgery in the two groups was statistically significant $\left(\chi^{2} 6.02, \mathrm{p}<0.02\right)$. There was no change in the parameters of brainstem function in group 1 patients after posterior surgery. There was an improvement, however, in eight patients (over 53\%) of group 2 , in the brainstem function parameters after posterior surgery.

Transoral excision of the odontoid was done in seven patients of group 2 after posterior surgery (table 3 ). In four of these patients the SSEP had deteriorated after posterior surgery. Following odontoid excision all four patients significantly improved in N19-N9 IPL. Clinical or electrophysiological parameters of the spinal cord and brainstem function improved in six patients. Comparison of the evoked potentials before posterior surgery with those after odontoid excision revealed improvement in I-III IPL in four (over 57\%) patients and of N19 wave/N19-N9 IPL in one patient. Five patients improved in N13 wave/N13-N9 IPL (table 4). There was, however, a deterioration in the IPLs in two patients. One patient had some improvement in the pyramidal tract signs.

In two group 2 patients, the transoral excision of the odontoid was followed by posterior fixation. Both patients had improved neurologically after surgery, although there was a change in the SSEP or BAEP.

\section{Discussion}

In recent years evoked potentials are routinely

Table 3 Change in clinical and electrophysiological parameters following transoral odontoid excision after posterior surgery in group 2 patients $(n=7)$

\begin{tabular}{llll}
\hline & Improved & Worsened & Unchanged \\
\hline Brainstem parameters & 6 & 0 & 1 \\
Cranial N paresis & 2 & 0 & 1 \\
I-III IPL & 4 & 2 & 1 \\
III-V IPL & 0 & 0 & 7 \\
Spinal cord parameters & 6 & 0 & 1 \\
Wasting & 0 & 0 & 7 \\
N9 wave or N13-N9 IPL & 2 & 0 & 5 \\
Pyramidal signs & 5 & 0 & 2 \\
Posterior column signs & 2 & 0 & 0 \\
N19 wave or N19-N9 IPL & 4 & 0 & 3 \\
\hline
\end{tabular}

used in various neurological disorders. ${ }^{89}$ BAEP and SEPs are very good indicators of brainstem and spinal cord integrity. Surprisingly, however, these tests have not been widely used in the management of craniovertebral junction abnormalities. ${ }^{1011}$ Some authors have used these tests in rheumatoid atlantoaxial, sublaxation or even Chiari-malformations. ${ }^{12-14}$ This study is the only large prospective study of evoked potential in patients with congenital craniovertebral anomaly.

This study shows a high incidence (10 patients, $31 \%$ ) of BAEP abnormality in patients of congenital atlantoaxial dislocation. In nine of these patients there was an associated basilar invagination. The abnormality was restricted to the I-III interval in most of these patients which suggested an involvement of the pontomedullary region. The prolongation of the III-V interval in one patient suggested that the dysfunction of the brainstem could be as high as the pontomesencephalic region in patients with basilar invagination. Lower cranial nerve palsies in nine patients suggested dysfunction of the medulla. The dysfunction of the medulla, pontomedullary region and the pontomesencephalic region was significantly higher $(p<0.05)$ in patients of atlantoaxial dislocation with basilar invagination. After posterior surgery, the I-III IPL improved in eight patients (over 53\%) in group 2 . In two of these patients the cranial nerve paresis had also improved. This may be a result of partial or complete reduction of the dislocation decreasing the ventral compression on the medulla and the pons by the odontoid or as a result of increased space in the posterior fossa after posterior decompression. Surprisingly in seven patients who had transoral excision of the odontoid, there was improvement in BAEP in four and of cranial nerve paresis in two.

Table 4 Comparison of clinical and electrophysiological parameters before posterior surgery and after odontoid excision in group 2 patients $(n=7)$

\begin{tabular}{llll}
\hline & Improved & Worsened & Unchanged \\
\hline Brainstem parameters & 5 & 1 & 1 \\
Cranial N paresis & 1 & 0 & 1 \\
I-III IPL & 4 & 1 & 2 \\
III-V IPL & 0 & 0 & 7 \\
Spinal cord parameters & 5 & 2 & 0 \\
Wasting & 0 & 0 & 7 \\
N9 wave or N13-N9 IPL & 5 & 1 & 1 \\
Pyramidal signs & 1 & 0 & 6 \\
Posterior column signs & 0 & 0 & 7 \\
N19 wave or N19-N9 IPL & 1 & 2 & 4 \\
\hline
\end{tabular}


The manifestations of the spinal cord dysfunction were not significantly different in the two groups. In both groups the N19-N9 and N13-N9 intervals were involved. The abnormality in N13 wave/N19-N9 IPL in 28 patients suggested an involvement of the posterior horns of the lower cervical spinal cord. There is evidence that the N13 component is mainly a postsynaptic potential generated in the posterior horns at the level of $\mathrm{C} 4-\mathrm{C} 7$ segments. ${ }^{9}$ Wasting of upper limbs was present in 14 patients. Wadia et al $^{15}$ reported wasting in 12 of the 28 patients of craniovertebral anomalies. The involvement of spinal segments in craniovertebral anomalies has been attributed to anoxia of these segments and also due to vascular congestion resulting from compression at the foramen magnum. ${ }^{15} 16$ The improvement in the N13 wave/N13-N9 IPL in some patients after surgery may be related to partial correction of the above factors.

Twenty five patients had abnormal N19 wave or N19-N9 IPL. Posterior column signs, however, were present in only 22 patients. The involvement of the posterior column was more often subclinical in patients in group $1(50 \%)$. The distortion of the spinal cord in two planes (horizontal as well as vertical) in patients with associated basilar invagination may be responsible for a more severe involvement of the posterior column in group 2 patients.

Thirty patients had involvement of the pyramidal tract. In five patients pyramidal tract signs were present in the absence of clinical or electrophysiological involvement of the posterior columns. Direct ventral compression by the odontoid may result in predominant involvement of pyramidal tracts only. Pathological changes have been reported to occur more frequently in the lateral columns than in the posterior columns in craniovertebral anomalies. $^{15}$

The hazards of posterior approach are well documented in atlantoaxial dislocations. ${ }^{2417}$ The deterioration in the neurological status was significantly higher following posterior surgery in patients with basilar invagination. Eleven of the 12 patients who had deteriorated had worsening of N19 wave N19-N9 IPL and in six, posterior column sensations had worsened. Pyramidal tract signs had worsened in seven of the 12 patients. This suggests that both pyramidal tracts and posterior columns are susceptible to deterioration after posterior surgery. The electrophysiological or clinical improvement in six of the seven patients who had odontoid resection after posterior surgery suggests that the deterioration after posterior surgery was related to distortion or compression and not to operative trauma. Selman et $a l^{9}$ showed intraoperative improvement in SEP in one of the two patients with congenital atlantoaxial dislocation who had transoral odontoid excision after posterior fusion. An increase in ventral cervicomedullary compression by the cerebellum sagging and kinking the cervicomedullary region over the odontoid, following posterior decompression was documented by Menezes and Van Gilder ${ }^{17}$ on postoperative MRI in patients with basilar invagination and Arnold-Chiari type I malformation. This may be responsible for neurological deterioration after posterior surgery in some patients of atlantoaxial dislocation with basilar invagination. Only two patients had an odontoid excision followed by posterior fusion and this combination of procedures appear to be preferable to an odontoid excision carried out after posterior surgery, as an overall deterioration in the SSEP and the BAEP accompanied the latter procedure.

Our results indicate that there is impairment of somatosensory and lower cervical spinal function in congenital atlantoaxial dislocations. The brainstem function is impaired when there is an associated basilar invagination. The somatosensory conduction is frequently worsened by posterior surgery and may then be improved by odontoid excision. The brainstem function, in contrast, has a good potential for recovery. The combination of posterior surgery and odontoid excision lead to favourable shifts in the BAEP in $57 \%$ of the patients. To avoid the deterioration that occurs after posterior surgery in patients with basilar invagination, excision of the odontoid as the first procedure seems more rational when a combination of posterior fusion and odontoid resection is contemplated.

Presented in part at the 9th International Congress of Neurological Surgery, 8-13 October 1989, New Delhi.

We are grateful to $\mathrm{Mr}$ Bisht and Mr Vijay of the Electrophysiology laboratory for assistance in evoked potential recordings.

1 Stratford J. Myelopathy caused by atlantoaxial dislocation. f Neurosurg 1957;14:97-104.

2 Dastur DR, Wadia NH, Desai AD, Singh G. Medullospinal compression due to atlanto-axial dislocation and sudden haematomyelia during decompression. Pathology, pathogenesis and clinical correlation. Brain 1965;88:897-924.

3 Singh G. Congenital atlantoaxial dislocation. Neurology India 1976;24:69-76.

4 Crocard HA, Pazo JL, Ranford AO, Steens JM, Kendell B, Essigman WK. Transoral decompression and posterior fixation for rheumatoid atlantoaxial sublaxation. $\mathcal{f}$ Bone Fnt Surg 1986;68(B):350-6.

5 Chippa KH. Evoke potentials in clinical medicine. New Eng f Med 1982;306:1205-10.

6 Greenberg RP, Ducker TB. Evoked potentials in clinical Neurosciences-Review article. $\underset{f}{ }$ Neurosurg 1982; 56:1-18.

7 Starr A. Sensory evoked potentials in clinical disorders of the nervous system. Ann Rev Neurosci 1978;1:103-27.

8 Salazzo D, Bruni P. Brainstem auditory evoked potentials abnormalities in the subjects with craniovertebral malformation. Ital $\mathcal{F}$ Neurol Sci 1985;6(2):185-90.

9 Selman WR, Spetzler RF, Brown R. The use of intraoperative fluroscopy and spinal cord monitoring for transoral microsurgical odontoid resection. Clin Orthop Rel Res 1981;154:51-6.

10 Chippa KE. Brainstem auditory evoked potentials interpretation. In: Chippa KE, (eds.) Evoked potentials in
clinical medicine, New York: Raven press, 1984:144-90.

11 Desmedt JJE, Cheron C. Prevertebral (Oesophageal) recording of subcortical somatosensory evoked potentials in man. The spinal P13 component and dual nature of spinal generators. Electroenceph Clin Neurophysiol 1981;52:257-75.

12 Holliday PO, Pillsbury D, Kelly DL, Dilland R. Brainstem auditory evoked potentials in Arnold Chiari malformaauditory evoked potentials in Arnold Chiari malforma-
tion. Possible prognostic value and changes with surgical tion. Possible prognostic value and changes with
decompression. Neurosurgery $1985 ; 16: 48-52$.

13 Schoenhuber R, Bortolotti P, Malavasi P, et al. Neurophysiological assessment of somatosensory pathology in rheumatic patients with atlantoaxial sublaxation. Electromyog Clin Neurophysiol 1984;24:213-6.

14 Toolan G, Kniben shol M, Larssen SE. Somatosensory evoked potentials in rheumatoid cervical sublaxation. Sca f Rheumatol 1987;16:17-25.

15 Wadia NH. Myelopathy complicating congenital atlantoaxial dislocation. A study of 28 cases. Brain 1967 90:449-72.

16 Dastur DR. Pathology and pathogenesis of chronic myelopathy atlantoaxial dislocation, with operative or postperative or haematomyelia or other cord complications. Clin Expe Neurol 1979;16:9-25.

17 Menezes AH, Van Gilder JC. Transoral-transpharyngeal approach to craniocervical lesions. Ten years experience with 72 cases. $\mathcal{f}$ Neurosurg 1988;69:895-905. 